OUTP-98-75 P

\title{
Interaction potential in compact three-dimensional QED with mixed action
}

\author{
A. Kovner \\ Theoretical Physics, University of Oxford, 1 Keble Road, Oxford, OX1 3NP, United Kingdom \\ B. Svetitsky \\ School of Physics and Astronomy, Raymond and Beverly Sackler Faculty of Exact Sciences, \\ Tel Aviv University, 69978 Tel Aviv, Israel
}

\begin{abstract}
We use a variational wave function to calculate the energy of the interaction between external charges in the compact Abelian gauge theory in $2+1$ dimensions with mixed action. Our variational wave functions preserve the compact gauge invariance of the theory both in the vacuum and in the charged sectors. We find that a good estimate of the interaction energy is obtained only when we allow more variational parameters in the charged sector than in the vacuum sector. These extra parameters are the profile of an induced electric field. We find that the theory has a two-phase structure: When the charge-2 coupling is large and negative there is no mass gap in the theory and no confinement, while otherwise a mass gap is generated dynamically and the theory confines charges. The pure Wilson theory is in the confining phase.
\end{abstract}

\section{INTRODUCTION}

The Abelian gauge theory in three dimensions has long been considered the archetype of a confining field theory. Polyakov [1] demonstrated charge confinement with a classic analysis of the instanton plasma; the hope that the result would carry over to the instantons of Yang-Mills theory in four dimensions proved, unfortunately, to be unfounded [2]. In the hands of Banks, Myerson, and Kogut [3], the theory became an exemplar of lattice gauge theory, displaying a single, confining phase from the trivial limit of strong coupling down to the expected essential singularity at weak coupling. It was also a stepping stone to elucidation of the four-dimensional Abelian theory, which confines at strong coupling but

\footnotetext{
1 The corresponding demonstration for the four-dimensional non-Abelian theory still depends on numerical simulation 沺].
} 
contains only weakly interacting photons at weak coupling: The difference between the two phases is due to a condensate of magnetic monopoles at strong couping, closely related to the instantons of the three-dimensional theory. Subsequently, the Hamiltonian version of the theory became a laboratory for development of variational methods [5], providing in this formalism as well a specimen of nonperturbative confinement persisting down to weak coupling.

In recent years there has been a revival of interest in variational approaches to nonAbelian gauge theories. Several calculations based on different variational ansätze have appeared in the literature [6, 7]. The approach of [6], a gauge invariant generalization of the Gaussian (mean field) variational approximation, was applied to the Abelian theory in [8], where it is clear that the wave function is a generalization of that used in [5] long ago. In the Yang Mills theory [6], there emerges a nonperturbative infrared mass scale, which makes the behavior of low momentum gluon modes in the vacuum very different from that calculated in perturbation theory. The comparison of the ultraviolet properties of this variational wave function with weak coupling perturbation theory was extended in [9, 10.

The results presented in [6,9,10] are limited to the wave function of the vacuum state. The most interesting phenomenon in non-Abelian gauge theories, however, is confinement of fundamental charges. In a Lorentz invariant theory one could, in principle, calculate instead the vacuum average of a spacelike Wilson loop. In the Hamiltonian framework, however, Lorentz invariance is not explicit and most variational approaches will preserve it (at best) only approximately. The relation in the variational framework between the vacuum average of the spacelike Wilson loop and the energy of external charges is poorly understood. It is therefore all the more interesting to set up a variational calculation for the charged sector.

In some recent papers [11,12] it was suggested that the charged sector can be described by the vacuum wave function, modified only to satisfy the new Gauss' law constraint. It was conjectured that further modification of the wave function in the presence of the external charges is unnecessary. We will refer to this state as the minimally modified vacuum. (Its exact definition will be given in Section 3.) It is far from evident that this simple procedure gives a good estimate of the energy of external charges. It does of course give a variational upper bound, but the quality of this bound may be poor. External charges introduce via Gauss' law an electric flux that is not present in the vacuum. It may be necessary to introduce additional variational parameters to allow this flux to spread out in the way that is most favorable energetically.

The Abelian gauge theory in $2+1$ dimensions is an ideal theater in which to examine this issue. The authors of [5] calculated the energy of an external dipole in this theory with the prescription advocated in [11,12]. The result was that the energy of the dipole is indeed proportional to its length and the string tension was determined. The vacuum expectation value of the spacelike Wilson loop was calculated in the same approach in [8]. There it was found that the Wilson loop obeys an area law with a string tension consistent with the result of Polyakov [1].

Superficially the results of [5] and [8] appear consistent. A closer look, however, reveals important differences. First, the vacuum state of [8] incorporates a dynamically generated mass whereas the correlations of magnetic and electric fields in the vacuum state of [5] have power law decays. Second, the string tension calculated in [5] (from the energy of external charges) is parametrically smaller than the result of [8] (from the spacelike Wilson loop). 
Finally, a calculation of the spacelike Wilson loop with the wave function of [5] gives no area law. In the present paper we explain these discrepancies while providing a more general discussion of the calculation of the energy in the presence of external charges.

We also take this opportunity to extend the variational analysis to the theory with mixed action. We define the theory with the lattice Hamiltonian

$$
H=\frac{1}{2} a^{2} \sum E_{\mathbf{n} i}^{2}-\frac{1}{g^{2} a^{2}} \sum\left(\alpha \cos g a^{2} B_{\mathbf{n}}+\frac{\beta}{4} \cos 2 g a^{2} B_{\mathbf{n}}\right)
$$

Here $a$ is the lattice spacing and the sums are respectively over the links and plaquettes of the two-dimensional spatial lattice. We constrain the constants $\alpha$ and $\beta$ to satisfy

$$
\alpha+\beta=1
$$

so that in the weak coupling limit, upon formal expansion to lowest order in $g^{2}$, the Hamiltonian reduces to the standard free Hamiltonian of $2+1$ dimensional electrodynamics. We work in this weakly coupled regime, $g^{2} a \ll 1$, but go beyond perturbation theory with a variational wave function.

The mixed Hamiltonian provides a new parameter for the theory. It has long been believed that there is no non-confining phase in three dimensions, but generalized couplings along the lines of (1.1) have not been adequately explored. Here we discover that this simplest of confining theories yet contains a surprise: a second-order confinement-deconfinement phase transition in the parameter $\alpha$. We present in Section 2 a variational calculation that determines the best variational vacuum of the theory. For $\alpha<\frac{\pi^{2}}{4}$ the energy is minimized for a state with a nonvanishing "mass gap," meaning that the correlation functions of the electromagnetic field decay exponentially at large distances. We will refer to this phase as massive. (This vacuum state is the same as that discussed in [8].) For $\alpha>\frac{\pi^{2}}{4}$ no such mass gap is generated, and the correlation functions at large distances have a power law decay. This phase will be called massless in the following. (This state is the same as that considered in [5].) Note that the Wilson action $\alpha=1$ is in the massive phase.2]

We also calculate the expectation values of Wilson loops in the two phases. In the massive phase we find an area law for Wilson loops of arbitrary charge, with the string tension proportional to the square of the charge. In the massless phase the string tension vanishes and there is no area law.

In Section 3 we calculate the potential between two external charges. First we consider the minimally modified vacuum according to the recipe of [5, 11, 12]. We calculate the profile of the electric field and the energy of a dipole configuration. We find that the results of this calculation are inconsistent with what we would infer from the behavior of the spacelike Wilson loop. In the massive phase, where we found an area law, the electric field does not form a flux tube but rather takes the form of an ordinary, unconfined dipole field. (This is

\footnotetext{
${ }^{2}$ Morris [13] has studied the three-dimensional Abelian theory with generalized action and found phase transitions to phases with non-zero magnetic field. We have fixed $\alpha+\beta=1$ to prevent this in the weak-coupling limit we study. Our new, non-confining phase is unrelated to Morris'.
} 
so even when the distance between the charges is much larger than the dynamical mass in the vacuum.) In the massless phase, amusingly, the situation is reversed. Even though there is no area law, the field far from the charges is screened and decays faster than a dipole field (but still as a power).

The calculation of the energy gives the following results, again inconsistent with the results of Section 2. In the massless phase we reproduce the result of [5], a linear confining potential. In the massive phase the energy of the minimally modified state diverges logarithmically in the infrared.

These results convince us that the minimally modified state provides a poor upper bound for the energy of the external dipole. . $^{\text {. }}$

In Section 4 therefore we introduce additional variational parameters into the trial state. These parameters are natural in the framework of the Gaussian approximation and correspond to a classical electric field present in the variational state. Varying the energy with respect to this classical field, we recover the results expected on the basis of the calculation in Section 2. In the massive phase the best variational state has the structure of an electric flux tube. Its energy is infrared finite and proportional to the distance between the charges. The string tension is the same as that calculated in Section 2. In the massless phase the flux spreads out into a dipole field, and correspondingly the energy has no linear dependence on the dipole size.

Finally in Section 5 we discuss our results and their implications for a possible calculation of the string tension in non-Abelian gauge theories.

\section{THE VARIATIONAL VACUUM}

\section{A. The wave function}

For a weakly coupled theory we expect the vacuum wave function to be not too different from that of a free theory. We therefore use a gauge invariant projection of a Gaussian wave function, as discussed in detail in [8].

We present the formalism here in lattice notation, corresponding to the Hamiltonian (1.1). (We suppress the lattice spacing $a$ henceforth.) Define a vortex field $A_{\mathbf{n} i}^{V}$ that satisfies

$$
\left(\nabla \times \mathbf{A}^{V}\right)_{\mathbf{n}^{\prime}}=\frac{2 \pi}{g} \delta_{\mathbf{n}^{\prime}, 0}, \quad \nabla \cdot \mathbf{A}^{V}=0
$$

where $\mathbf{n}^{\prime}$ is a site of the dual lattice. This is the vector potential corresponding to a magnetic field that is zero everywhere except at $\mathbf{n}^{\prime}=0$, where it takes the value $\frac{2 \pi}{g}$. We demand that our variational wave function $\psi[\mathbf{A}]$ be invariant under shifts $\mathbf{A} \rightarrow \mathbf{A}+\mathbf{A}^{V}$, which is an expression of the periodicity of $H$ under $B \rightarrow B+\frac{2 \pi}{g}$. We also demand gauge invariance

\footnotetext{
${ }^{3}$ Ben-Menahem [14] noted that the massless wave functions of [5] gave correlations inconsistent with the Euclidean analysis [1, 3 , and proposed a massive propagator to correct the discrepancy.
} 
of the wave function. Hence we define a shifted field, shifted by a gauge transformation $\phi_{\mathbf{n}}$ and by a vortex distribution $m_{\mathbf{n}^{\prime}}$,

$$
\mathbf{A}_{\mathbf{n}}^{(\phi, m)}=\mathbf{A}_{\mathbf{n}}-(\nabla \phi)_{\mathbf{n}}-\sum_{\mathbf{n}^{\prime}} m_{\mathbf{n}^{\prime}} \mathbf{A}^{V}\left(\mathbf{n}-\mathbf{n}^{\prime}\right)
$$

or, for short,

$$
A^{(\phi, m)}=A-\nabla \phi-A^{V} \cdot m
$$

Then the gauge invariant and periodic trial wave function is

$$
\psi[\mathbf{A}]=\sum_{\left\{m_{\mathbf{n}^{\prime}}\right\}} \int\left[d \phi_{\mathbf{n}}\right] \exp \left[-\frac{1}{2} \sum_{\mathbf{r}, \mathbf{s}} A_{\mathbf{r} i}^{(\phi, m)} G^{-1}(\mathbf{r}-\mathbf{s}) A_{\mathbf{s} i}^{(\phi, m)}\right] .
$$

Under a gauge transformation,

$$
\psi[\mathbf{A}+\nabla \lambda]=\psi[\mathbf{A}]
$$

since $\lambda$ can be absorbed in a shift in $\phi$. The simple rotational structure of $G_{i j}=\delta_{i j} G$ that appears in the variational wave function (2.4) is consistent with perturbation theory, as discussed in [6]. In this paper we take $G(x)$ to be a real function. We now proceed to calculation of expectation values in the trial state (2.4) and the minimization of the vacuum energy.

\section{B. Normalization integral}

The normalization of $|\psi\rangle$ is

$$
Z \equiv\langle\psi \mid \psi\rangle=\sum_{\left\{m, m^{\prime}\right\}} \int[d \phi]\left[d \phi^{\prime}\right][d \mathbf{A}] e^{-\frac{1}{2} A^{(\phi, m)} G^{-1} A^{(\phi, m)}} e^{-\frac{1}{2} A^{\left(\phi^{\prime}, m^{\prime}\right)} G^{-1} A^{\left(\phi^{\prime}, m^{\prime}\right)}} .
$$

We shift $\mathbf{A}$ by $\nabla \phi^{\prime}+\mathbf{A}^{V} \cdot m^{\prime}$, and absorb the shift into $\phi$ and $m$, giving

$$
Z=\sum_{\{m\}} \int[d \phi][d \mathbf{A}] e^{-\frac{1}{2} A^{(\phi, m)} G^{-1} A^{(\phi, m)}} e^{-\frac{1}{2} A G^{-1} A} .
$$

Now we combine the exponents according to

$$
A^{(\phi, m)} G^{-1} A^{(\phi, m)}+A G^{-1} A=2 A^{(\phi / 2, m / 2)} G^{-1} A^{(\phi / 2, m / 2)}+\frac{1}{2} S(\phi, m) G^{-1} S(\phi, m)
$$

where

$$
S \equiv \nabla \phi+A^{V} \cdot m
$$

The first term in $\mathbf{S}$ has zero curl while the second is divergenceless. Furthermore, $G^{-1}$ is translation invariant and proportional to the unit matrix. Thus $S G^{-1} S$ has no cross terms 
between $m$ and $\phi$. We now shift $\mathbf{A}$ by $\nabla \phi / 2+\mathbf{A}^{V} \cdot m / 2$, and all the fields decouple. We have then

$$
Z=Z_{A} Z_{\phi} Z_{v}
$$

where

$$
\begin{gathered}
Z_{A}=\operatorname{det} \pi G \\
Z_{\phi}=\int[d \phi] e^{-\frac{1}{4} \nabla \phi \cdot G^{-1} \cdot \nabla \phi} \\
=\left(\operatorname{det} 4 \pi \frac{1}{\nabla^{2}} G\right)^{1 / 2}, \\
Z_{v}=\sum_{\left\{m_{\mathbf{n}^{\prime}}\right\}} \exp \left[-\frac{1}{4 g^{2}} \sum_{\mathbf{r}^{\prime}, \mathbf{s}^{\prime}} m_{\mathbf{r}^{\prime}} D\left(\mathbf{r}^{\prime}-\mathbf{s}^{\prime}\right) m_{\mathbf{s}^{\prime}}\right] .
\end{gathered}
$$

$Z_{v}$ is the vortex partition function, with the vortex-vortex interaction $D$ given by

$$
D\left(\mathbf{r}^{\prime}-\mathbf{s}^{\prime}\right)=g^{2} \sum_{\mathbf{r}, \mathbf{s}} \mathbf{A}^{V}\left(\mathbf{r}-\mathbf{r}^{\prime}\right) \cdot G^{-1}(\mathbf{r}-\mathbf{s}) \mathbf{A}^{V}\left(\mathbf{s}-\mathbf{s}^{\prime}\right)
$$

The explicit solution of $(2.1)$ is

$$
A_{i}^{V}(\mathbf{n})=-\frac{2 \pi}{g} \epsilon_{i j}\left(\frac{\partial_{j}}{\nabla^{2}} \delta_{\mathbf{n}^{\prime}, 0}\right)_{\mathbf{n}}
$$

So

$$
D=-g^{2} A^{V} G^{-1} A^{V}=-4 \pi^{2} \frac{1}{\nabla^{2}} G^{-1}
$$

We can split off the $\mathbf{r}^{\prime}=\mathbf{s}^{\prime}$ terms in (2.13) and write

$$
Z_{v}=\sum_{\left\{m_{\mathbf{n}^{\prime}}\right\}} \exp \left[-\frac{1}{4 g^{2}} \sum_{\mathbf{r}^{\prime} \neq \mathbf{s}^{\prime}} m_{\mathbf{r}^{\prime}} D\left(\mathbf{r}^{\prime}-\mathbf{s}^{\prime}\right) m_{\mathbf{s}^{\prime}}\right] \prod_{\mathbf{r}^{\prime}} z^{m_{\mathbf{r}^{\prime}}^{2}}
$$

where we have defined the vortex fugacity

$$
z=e^{-\frac{1}{4 g^{2}} D(0)}
$$

In the interest of clarity, we adopt henceforth a continuum notation, indicating the ultraviolet cutoff $a$ only where necessary. Moreover, since the interesting physics comes from the infrared, lattice effects can be approximated by a momentum-space cutoff $\Lambda=a^{-1}$, which can simplify formulas further.

The variational function $G$ appears both in the vortex-vortex potential and in the vortex fugacity. We expect the UV behavior of $G$ to be the same as in the free theory, viz., 


$$
G^{-1}(k) \sim k
$$

so

$$
\begin{aligned}
D(0) & \sim \int^{\Lambda} \frac{d^{2} k}{(2 \pi)^{2}} \frac{4 \pi^{2}}{k^{2}} G^{-1}(k) \\
& \sim 2 \pi \Lambda
\end{aligned}
$$

and thus

$$
z \sim e^{-\frac{\pi}{2} \frac{1}{g^{2} a}}
$$

In the weak coupling region we have $z \ll 1$, justifying a restriction to $m=0, \pm 1$ in $(2.13)$ and (2.17).

\section{Expectation values}

We calculate correlation functions of $m$ as in [8] via a duality transformation. We add an $i J \cdot m$ term to the exponent in (2.13) and use the formula

$$
e^{-\frac{1}{4 g^{2}} m \cdot D \cdot m}=\mathrm{const} \int[d \chi] e^{-g^{2} \chi \cdot D^{-1} \cdot \chi} e^{i \chi \cdot m}
$$

to obtain

$$
Z_{v}=\int[d \chi] e^{-g^{2} \chi \cdot D^{-1} \cdot \chi} \prod_{\mathbf{n}}\left[1+2 \cos \left(\chi_{\mathbf{n}}+J_{\mathbf{n}}\right)\right]
$$

Noting that

$$
\cos (\chi+J)=\langle\cos \chi\rangle_{0}: \cos (\chi+J):=z: \cos (\chi+J):
$$

we have

$$
\begin{aligned}
Z_{v} & =\int[d \chi] e^{-g^{2} \chi \cdot D^{-1} \cdot \chi} \prod[1+2 z: \cos (\chi+J):] \\
& \simeq \int D \chi \exp \left[-g^{2} \chi D^{-1} \chi+2 z \int d^{2} x: \cos (\chi(x)+J(x)):\right]
\end{aligned}
$$

in continuum notation. Correspondingly [8],

$$
\langle m(x) m(y)\rangle=2 g^{2} D^{-1}(x-y)-4 g^{4}\left\langle D^{-1} \chi(x) D^{-1} \chi(y)\right\rangle .
$$

The propagator of $\chi$ is easily calculated. To first order in $z$, the only contribution comes from the tadpole diagrams, which have already been subtracted in (2.26). Therefore

\footnotetext{
${ }^{4}$ The normal ordering is performed relative to the free theory defined by the quadratic action in (2.24).
} 


$$
\begin{aligned}
\int d^{2} x e^{i k x}\langle\chi(x) \chi(0)\rangle & =\frac{1}{2 g^{2} D^{-1}(k)+2 z} \\
& =\frac{D(k)}{2 g^{2}}-z \frac{D^{2}(k)}{2 g^{4}}+O\left(z^{2}\right)
\end{aligned}
$$

The correlation function of the vortex density is then

$$
K(k) \equiv \int d^{2} x e^{i k x}\langle m(x) m(0)\rangle=2 z+O\left(z^{2}\right)
$$

which in this approximation does not depend on momentum. The $k$ dependence will appear in the higher orders in $z$.

Now we are ready to calculate the expectation value of the Hamiltonian (1.1). The calculation of the electric part of the energy is identical to that in [8]. Using the definition (2.4) we calculate

$$
\begin{aligned}
V^{-1}\left\langle\int E^{2} d^{2} x\right\rangle & =-\frac{1}{V}\left\langle\psi\left|\sum_{\mathbf{n}, i} \frac{\partial^{2}}{\partial A_{\mathbf{n}, i}^{2}}\right| \psi\right\rangle \\
& =\frac{1}{2} \int \frac{d^{2} k}{(2 \pi)^{2}} G^{-1}(k)-\frac{\pi^{2}}{g^{2}} \int \frac{d^{2} k}{(2 \pi)^{2}} k^{-2} G^{-2}(k) K(k) \\
& =\frac{1}{2} \int \frac{d^{2} k}{(2 \pi)^{2}} G^{-1}(k)-\frac{2 \pi^{2}}{g^{2}} z \int \frac{d^{2} k}{(2 \pi)^{2}} k^{-2} G^{-2}(k)
\end{aligned}
$$

The magnetic part is easily calculated since it has an exponential form and therefore with our trial wave function leads to a simple Gaussian integral. We find

$$
\left\langle e^{i n g B_{\mathbf{n}}}\right\rangle=\exp \left[-\frac{1}{4} n^{2} g^{2} \int \frac{d^{2} k}{(2 \pi)^{2}} k^{2} G(k)\right]\left\langle e^{i n \pi m_{\mathbf{n}}}\right\rangle \text {. }
$$

The second factor is due to the vortices, and is different from unity only for odd values of $n$. Using (2.26) we find easily that $\left\langle e^{i \pi m}\right\rangle=e^{-4 z}$. Expanding to leading order in $g^{2}$ and $z$,

$$
\left\langle-\frac{1}{g^{2}}\left(\alpha \cos g B+\frac{1-\alpha}{4} \cos 2 g B\right)\right\rangle=\frac{1}{4} \int \frac{d^{2} k}{(2 \pi)^{2}} k^{2} G(k)+\frac{4 \alpha}{g^{2}} z .
$$

(We have dropped an additive constant.) Finally, the expression for the variational vacuum expectation value of the energy is

$$
\frac{1}{V}\langle H\rangle=\frac{1}{4} \int \frac{d^{2} k}{(2 \pi)^{2}}\left[G^{-1}(k)+k^{2} G(k)-\frac{4 \pi^{2}}{g^{2}} z\left(k^{-2} G^{-2}(k)-\frac{4}{\pi^{2}} \alpha\right)\right] .
$$

\section{Determination of the ground state}

The expression (2.34) has to be minimized with respect to $G$. From equation (2.18) and (2.20) we find 


$$
\frac{\delta z}{\delta G(k)}=\frac{1}{4 g^{2}} k^{-2} G^{-2}(k) z
$$

The variation of (2.34) gives

$$
k^{2}-G^{-2}(k)=\frac{4 \pi^{4}}{g^{4}} z k^{-2} G^{-2}(k) \int \frac{d^{2} p}{(2 \pi)^{2}}\left[p^{-2} G^{-2}(p)-\frac{4}{\pi^{2}} \alpha\right] .
$$

Eq. (2.36) has the solution

$$
G^{-2}(k)=\frac{k^{4}}{k^{2}+m^{2}}
$$

where

$$
m^{2}=\frac{4 \pi^{4}}{g^{4}} z \int \frac{d^{2} k}{(2 \pi)^{2}}\left[k^{-2} G^{-2}(k)-\frac{4}{\pi^{2}} \alpha\right] .
$$

The main contribution to the integral in the gap equation (2.38) comes from momenta $k^{2} \gg m^{2}$. For these momenta $k^{2} G^{-2}(k)=1$. We therefore see that (2.38) has a nontrivial solution when $\alpha<\frac{\pi^{2}}{4}$. Using equations (2.18), (2.20), and (2.37) we obtain

$$
m^{2}=\frac{4 \pi^{4}}{g^{4}} \exp \left(-\frac{\pi^{2}}{g^{2}} \int \frac{d^{2} p}{(2 \pi)^{2}} \frac{1}{\sqrt{p^{2}+m^{2}}}\right) \int \frac{d^{2} k}{(2 \pi)^{2}}\left[\frac{k^{2}}{k^{2}+m^{2}}-\frac{4}{\pi^{2}} \alpha\right]
$$

which for $g^{2} \ll 1$ can be simplified to [cf. (2.22)]

$$
m^{2}=4 \pi^{2} \frac{\left(\pi^{2}-4 \alpha\right)}{g^{4}} \exp \left(-\frac{\pi}{2 g^{2}}\right) .
$$

$m$ is the mass gap of the theory, in the sense that it is the inverse of the spatial correlation length. Calculating, for example, the propagator of magnetic field, we find

$$
\left\langle e^{i g B_{\mathbf{m}}} e^{-i g B_{\mathbf{n}}}\right\rangle=\left|\left\langle e^{i g B}\right\rangle\right|^{2} e^{\frac{g^{2}}{2} \nabla^{2} G(\mathbf{m}-\mathbf{n})},
$$

and at large distances (neglecting power-like prefactors),

$$
\nabla^{2} G(x)=-\int \frac{d^{2} k}{(2 \pi)^{2}}\left(k^{2}+m^{2}\right)^{1 / 2} e^{i \mathbf{k} \cdot \mathbf{x}} \sim e^{-m x} .
$$

This dynamically generated mass is Polyakov's result [1], and is missing from [5].

For $\alpha>\frac{\pi^{2}}{4}$, 2.38) has no real solution for $m$. In this case the energy is minimized at the endpoint of the variational parameter range, $m^{2}=0$. We conclude that in the Gaussian variational approximation, mixed action $\mathrm{QED}_{3}$ has a phase transition at $\alpha_{c}=\frac{\pi^{2}}{4}$. The mass

\footnotetext{
${ }^{5}$ We have dropped a term $-\frac{8 \pi^{2}}{g^{2}} z k^{-2} G^{-3}(k)$ from the right hand side of (2.36) is smaller by a factor of $\frac{g^{2} k}{\Lambda^{2}}$ than the term retained, assuming $G \sim k^{-1}$ at large $k$.
} 
gap vanishes continuously at the critical point while $(\partial m / \partial \alpha)_{\alpha_{c}^{-}} \neq 0$. The phase transition therefore appears to be second order.

The energy density (2.34) contains ultraviolet contributions. The vacuum energy density in (almost) any field theory is ultraviolet divergent; thus it should diverge for any variational ansatz and indeed (2.34) contains such a divergence. The crucial question for us is whether the leading ultraviolet divergent terms depend on the variational parameter. This would indicate that the parameter is introduced in a way that directly affects the UV properties of the state. In other words such a dependence would mean that one is dealing with a set of states for which even the UV part of energy is not minimized yet. Such a variation would have little to say about IR physics.

Examining (2.34) we see that the leading UV divergent terms are independent of the variational mass $m$. The UV behaviour of $G$ is determined (in both phases) independently of $m$ and is consistent with free field theory in the high momentum limit. (This indeed should be the case since the $3 \mathrm{~d} \mathrm{U}(1)$ gauge theory is superrenormalizable and its UV physics is governed by a fixed point at zero coupling.) Nontrivial information about the IR physics resides not in the leading terms in the energy but in the subleading ones. This makes us confident that the ultraviolet and infrared physics have indeed been separated in a clean way in our variational calculation.

\section{E. Spacelike Wilson loops}

Let us see whether charges are confined in the two phases. To this end we calculate the vacuum expectation value of the spacelike Wilson loop,

$$
W_{l}[C]=\left\langle\exp \left(i \lg \oint_{C} \mathbf{A} \cdot d \mathbf{x}\right)\right\rangle=\left\langle\exp \left(i l g \int_{S} B d S\right)\right\rangle
$$

where $l$ is an integer and the integral is over the area $S$ bounded by the loop $C$. We have

$$
W_{l}[C]=\left\langle\prod_{S} e^{i l \pi m_{\mathbf{n}}}\right\rangle Z_{A}^{-1} \int D \mathbf{A} \exp \left(-A G^{-1} A+i l g \int_{S} B d S\right)
$$

The second factor is a Gaussian integral, which gives the factor

$$
W_{A}=\exp \left(\frac{l^{2} g^{2}}{4} \int_{S} d^{2} x \int_{S} d^{2} y \nabla^{2} G(\mathbf{x}-\mathbf{y})\right) .
$$

In the limit of large $S$ the leading behavior of the exponent is

$$
-\frac{l^{2}}{4} g^{2} S \lim _{k \rightarrow 0} k^{2} G(k)=-\frac{l^{2}}{4} g^{2} m S \text {. }
$$

\footnotetext{
${ }^{6}$ The value of the critical coupling $\alpha_{c}=\frac{\pi^{2}}{4}$ should not be taken too literally, since $G$ is modified from its continuum form close to the boundaries of the Brillouin zone. It is however clear that the phase transition is present in any cutoff scheme, at least in the present approximation.
} 
In the massive phase this gives an area law with the string tension!

$$
\sigma=\frac{l^{2}}{4} g^{2} m
$$

In the massless phase this factor does not produce an area law.

The first factor in equation (2.44) is different from unity only for odd $l$. It can be easily calculated to be

$$
W_{v} \equiv\left\langle\prod_{S} e^{i l \pi m_{\mathbf{n}}}\right\rangle=\int D \chi \exp \left[-g^{2} \chi D^{-1} \chi+2 z \int d^{2} x: \cos (\chi(x)-\alpha(x)):\right],
$$

where $\alpha(x)$ vanishes for $x$ outside the loop and is equal to $\pi$ for $x$ inside the loop. Recall that, from (2.16),

$$
D(k)=4 \pi^{2} k^{-2} G^{-1}(k)=4 \pi^{2}\left(k^{2}+m^{2}\right)^{-1 / 2} .
$$

At weak coupling we expand around a classical minimum of the exponent. In the massive phase, $\alpha<\frac{\pi^{2}}{4}$, the inverse propagator $D^{-1}$ is nonzero at zero momentum and thus it dictates the leading order solution $\chi(x)=0$. For this solution we find

$$
W_{v}=e^{-4 z S} .
$$

This is a sub-leading correction to the string tension (2.47), since $g^{2} m \sim e^{-\pi / 4 g^{2}}$ and $z \propto$ $m^{2} \ll g^{2} m$.

In the massless phase the situation is different. Here the kinetic term of the dual field $\chi$ vanishes at zero momentum, $D^{-1}(0)=0$. Any constant value of $\chi$ is therefore compatible with the kinetic term. The classical configuration that minimizes the vortex action in (2.48) for large area $\left(S \gg z^{-1}\right)$ is $\chi=0$ outside the area enclosed by the loop and $\chi=\pi$ well inside the area. The field interpolates between these two values in a layer of thickness $z^{-1}$ in the neighborhood of the loop? The action for this configuration clearly is proportional to the perimeter. Thus in the massless phase the string tension vanishes altogether.

The spacelike Wilson loop tells us that the massive phase is confining with the string tension related in the expected way to the dynamically generated scale, $\sigma \propto g^{2} m$. The massless phase does not exhibit an area law for spacelike Wilson loops and we therefore expect that the static charges in this phase are not confined.

\footnotetext{
${ }^{7}$ As was shown in [15] the dependence on $l$ in this formula is incorrect. The correct result is $\sigma \propto l$ rather than $\sigma \propto l^{2}$. For multiply charged Wilson loops the nonlinearities of the compact theory are important and the Gaussian variational ansatz may be inadequate. This point is peripheral to the present paper and hence we will not pursue it.

${ }^{8}$ In the massive phase this classical configuration possesses an enormous action $\sim g^{2} m S$, proportional to the area, since the kinetic term $D^{-1}$ does not vanish at zero momentum. The leading contribution to the Wilson loop therefore comes from configurations with $\chi=0$ well inside the loop as stated above.
} 


\section{THE MINIMALLY MODIFIED STATE}

As we mentioned in the Introduction, in a Lorentz invariant theory the string tension extracted from the spacelike Wilson loop should be the same string tension that determines the strength of the linear potential between two static charges. Lorentz symmetry, however, is not maintained in a variational calculation. Moreover, the theory we are studying is defined with an explicit ultraviolet regulator that itself breaks Lorentz invariance. It is thus interesting to perform a separate calculation of the potential between external charges.

\section{A. The wave function}

In the charged sector the gauge invariance condition (2.5) is replaced by

$$
\psi[\mathbf{A}+\nabla \lambda]=\psi[\mathbf{A}] \exp \left(i g \sum_{\mathbf{n}} \rho_{\mathbf{n}} \lambda_{\mathbf{n}}\right)
$$

where $\rho_{\mathbf{n}}$ is a fixed, integer background charge distribution. (We have in mind a wellseparated dipole.) A wave function that satisfies (3.1) is

$$
\psi[\mathbf{A}]=\sum_{\left\{m_{\mathbf{n}^{\prime}}\right\}} \int\left[d \phi_{\mathbf{n}}\right] \exp \left[-\frac{1}{2} \sum_{\mathbf{r}, \mathbf{s}} A_{\mathbf{r} i}^{(\phi, m)} G^{-1}(\mathbf{r}-\mathbf{s}) A_{\mathbf{s} i}^{(\phi, m)}\right] \exp \left(i g \sum_{\mathbf{n}} \rho_{\mathbf{n}} \phi_{\mathbf{n}}\right) .
$$

The shifted field in (3.2) is defined along the lines of (2.3),

$$
A^{(\phi, m)}=A-\nabla \phi-A^{S} \cdot m .
$$

but now it is necessary to define the vortex field $A^{S}$ in a singular gauge. Like $A^{V}$, the field $A^{S}$ satisfies

$$
\left(\nabla \times \mathbf{A}^{S}\right)_{\mathbf{n}^{\prime}}=\frac{2 \pi}{g} \delta_{\mathbf{n}^{\prime}, 0}
$$

but while $A^{V}$ is divergenceless we take for $A^{S}$ the solution where $A$ is non-zero on the links dual to a string extending from 0 (the plaquette of the vortex) to $x=+\infty$,

$$
\begin{aligned}
& A_{\mathbf{n} x}^{S}=0 \\
& A_{\mathbf{n} y}^{S}= \begin{cases}\frac{2 \pi}{g} & \text { for } n_{x}>0, n_{y}=0 \\
0 & \text { otherwise }\end{cases}
\end{aligned}
$$

Our reason for using $A^{S}$ rather than $A^{V}$ in (3.2) is one of locality. A dipole can be created in the vacuum (2.4) by the string operator $\exp \left[i g \sum_{\mathbf{x}}^{\mathbf{y}} A(\mathbf{z})\right]$, which places sources at $\mathbf{x}$ and $\mathbf{y}$. This operator creates a string of electric flux taking integer values along the string, the most local way of preserving Gauss' Law when creating a dipole. A shift of $\mathbf{A}$ by $A^{S}$ commutes with this string operator, but a shift by $A^{V}$ does not. Shifting by $A^{V}$ will create a non-local, transverse electric field with fractional flux in addition to the string. In this light it appears unavoidable that the introduction of dynamical charges will immediately lead to a nonlocal and non-Lorentz-invariant theory. For more on this point see [5]. 
Another way of looking at it is to note that the difference between $A^{V}$ and $A^{S}$ can be absorbed by a shift in the integration variable $\phi_{\mathbf{r}}$ by $\frac{1}{g} \theta\left(\mathbf{r}-\mathbf{r}^{\prime}\right) m_{\mathbf{r}^{\prime}}$, where $\theta\left(\mathbf{r}-\mathbf{r}^{\prime}\right)$ is the angle that the vector $\mathbf{r}-\mathbf{r}^{\prime}$ makes with the $x$ axis. Using $A^{V}$ would therefore lead to an extra phase factor $\exp \left[i \sum_{\mathbf{r}, \mathbf{r}^{\prime}} \rho_{\mathbf{r}} \theta\left(\mathbf{r}-\mathbf{r}^{\prime}\right) m_{\mathbf{r}^{\prime}}\right]$ in the integral in (3.2). Under a shift of vortex density $m$ this function is not invariant, but rather acquires a phase proportional to the charge density. The shift of vortex density can be viewed as a kind of large gauge transformation [8] and therefore this wave function belongs to a sector of the Hilbert space with a position-dependent " $\theta$ angle." It is hard to imagine how this sector can define a local theory, especially in the presence of dynamical charges.

Since $A^{S}$ differs from $A^{V}$ by a gauge transformation, it can be used interchangeably with it in the vacuum wave function (2.4). The distinction is only meaningful in the charged sector, where it leads to a new vortex-charge interaction [see (3.9) below].

Taking $G$ to be the same function as was used in Section $\llbracket$ in the vacuum sector, this is the minimally modified state used by [5, [1, 12].

\section{B. Normalization and expectation values}

The normalization factor for the wave function (3.2) is

$$
Z=Z_{A} Z_{\phi}[\rho] Z_{v}[\rho]
$$

with

$$
\begin{aligned}
Z_{A} & =\operatorname{det} \pi G \\
Z_{\phi}[\rho] & =\left(\operatorname{det} 4 \pi \frac{1}{\nabla^{2}} G\right)^{1 / 2} \exp \left(-g^{2} \rho \cdot \frac{1}{\nabla^{2}} G \cdot \rho\right), \\
Z_{v}[\rho] & =\sum_{\left\{m_{\mathbf{n}^{\prime}}\right\}} \exp \left[-\frac{1}{4 g^{2}} \sum_{\mathbf{r}^{\prime}, \mathbf{s}^{\prime}} m_{\mathbf{r}^{\prime}} D\left(\mathbf{r}^{\prime}-\mathbf{s}^{\prime}\right) m_{\mathbf{s}^{\prime}}\right] \exp \left(-i \sum_{\mathbf{r}^{\prime}} h_{\mathbf{r}^{\prime}} m_{\mathbf{r}^{\prime}}\right) .
\end{aligned}
$$

The new ingredient in (3.9) is a vortex-charge interaction,

$$
\sum_{\mathbf{r}^{\prime}} h_{\mathbf{r}^{\prime}} m_{\mathbf{r}^{\prime}} \equiv \sum_{\mathbf{r} \mathbf{r}^{\prime}} \rho_{\mathbf{r}} \theta\left(\mathbf{r}-\mathbf{r}^{\prime}\right) m_{\mathbf{r}^{\prime}}
$$

The vortex interaction potential $D$ is again given by (2.16).

First let us calculate the expectation value of the electric field in this state. Straightforward algebra gives

$$
\left\langle E_{i}\right\rangle=\left\langle i \frac{\partial}{\partial A_{i}}\right\rangle=g \frac{\partial_{i}}{\nabla^{2}} \rho-\frac{i \pi}{g} G^{-1} \frac{\epsilon_{i j} \partial_{j}}{\nabla^{2}}\langle m\rangle
$$

The first term on the right hand side is the longitudinal field that gives the Coulomb interaction between the charges in non-compact electrodynamics. The second term, entirely due to the compactness of the theory, is purely transverse as it must be in order not to spoil Gauss' law. 
The calculation of the energy follows the same lines as the analogous calculation discussed in some detail in the previous section. For the kinetic term [cf. (2.30)],

$$
\begin{aligned}
\left\langle\int E^{2} d^{2} x\right\rangle=\frac{V}{2} \int & \frac{d^{2} k}{(2 \pi)^{2}} G^{-1}(k)-g^{2} \int d^{2} x \rho \frac{1}{\nabla^{2}} \rho \\
& +\frac{\pi^{2}}{g^{2}} \int d^{2} x d^{2} y \partial^{-2} G^{-2}(x-y)\langle m(x) m(y)\rangle
\end{aligned}
$$

while the potential term is

$$
\left\langle-\frac{1}{g^{2}}\left(\alpha \cos g B+\frac{1-\alpha}{4} \cos 2 g B\right)\right\rangle=\frac{1}{4} \int \frac{d^{2} k}{(2 \pi)^{2}} k^{2} G(k)-\frac{\alpha}{g^{2}}\left(\operatorname{Re}\left\langle e^{i \pi m}\right\rangle-1\right) .
$$

Putting these together we obtain for the energy of the minimally modified state

$$
\begin{aligned}
\langle H\rangle=\frac{V}{4} \int & \frac{d^{2} k}{(2 \pi)^{2}}\left[G^{-1}(k)+k^{2} G(k)\right]-\frac{g^{2}}{2} \int d^{2} x \rho \frac{1}{\nabla^{2}} \rho \\
& +\frac{\pi^{2}}{2 g^{2}} \int d^{2} x d^{2} y \partial^{-2} G^{-2}(x-y)\langle m(x) m(y)\rangle \\
& -\frac{\alpha}{g^{2}} \int d^{2} x\left(\operatorname{Re}\left\langle e^{i \pi m(x)}\right\rangle-1\right) .
\end{aligned}
$$

To calculate correlation functions of the vorticity $m$, we again introduce the dual field $\chi$ as in (2.23) and obtain the relations

$$
\begin{aligned}
\langle m\rangle & =-2 i g^{2} D^{-1}\langle\chi\rangle \\
\langle m(x) m(y)\rangle & =2 g^{2} D^{-1}-4 g^{4}\left\langle D^{-1} \chi(x) D^{-1} \chi(y)\right\rangle,
\end{aligned}
$$

where the averages of the dual field $\chi$ are calculated with the partition function

$$
Z_{v}=\int D \chi \exp \left[-g^{2} \chi D^{-1} \chi+2 z \int d^{2} x: \cos (\chi(x)-\theta \rho):\right] .
$$

[The notation $\theta \rho$ represents the convolution of the source distribution $\rho(\mathbf{r})$ with $\theta\left(\mathbf{r}-\mathbf{r}^{\prime}\right)$.] In the weak coupling ( small $z$ ) regime we solve the classical equation of motion,

$$
g^{2} D^{-1} \chi+z \sin (\chi-\theta \rho)=0
$$

We consider the massive $\left(\alpha<\alpha_{c}\right)$ and massless $\left(\alpha>\alpha_{c}\right)$ cases separately.

\section{Field profile and energy in the massive phase}

First let us take $\alpha<\alpha_{c}$. In this case the variational propagator $G$ is massive. This mass is large compared to $z$ since $z \propto m^{2} \ll g^{2} m$. Recall that $D^{-1} \sim \sqrt{k^{2}+m^{2}}$. The classical equation (3.17) can then be solved in perturbation theory in $\frac{z}{g^{2} m}$. To first order in $z$ we find

$$
\chi=\frac{z D}{g^{2}} \sin (\theta \rho)
$$


and thus

$$
\begin{aligned}
\langle m(x)\rangle & =-2 i z \sin (\theta \rho(x)) \\
\langle m(x) m(y)\rangle_{\text {connected }} & =2 z \cos (\theta \rho(x)) \delta^{2}(x-y) \\
\left\langle e^{i \pi m(x)}\right\rangle & =\exp [-4 z \cos (\theta \rho(x))]
\end{aligned}
$$

Our first observation is that with this average value of the vortex density the electric field far away from the charges is not screened, but rather decays the same way as in the non-compact theory. To see this let us rewrite the expression for the electric field in terms of the dual field $\chi$,

$$
\left\langle E_{i}\right\rangle=g \frac{\partial_{i}}{\nabla^{2}} \rho+\frac{g}{2 \pi} \epsilon_{i j} \partial_{j}\langle\chi\rangle .
$$

In order for the electric field to be screened away from the charges, the first and second terms must cancel. For this to happen the dual field $\chi$ must behave asymptotically as

$$
\chi(x) \rightarrow \theta \rho(x) \text { as } x \rightarrow \infty .
$$

To find the large-distance behavior of the solution (3.18) we take for $D\left(k^{2}\right)$ its value at zero momentum, $D(0)=\left(4 \pi^{2} m\right)^{-1}$. We then find

$$
\chi(x \rightarrow \infty) \sim \frac{z}{g^{2} m} \sin (\theta \rho(x))
$$

which is much smaller than required by (3.21), so there is no cancellation of the long range part of the electric field.

Technically it is clear why we get the estimate (3.22) rather than (3.21) for the asymptotic behavior of the classical solution. If we were to minimize only the potential term in the dual action, (3.21) would be the result. In the massive phase, however, the kinetic term $\chi D^{-1} \chi$ is the leading term for configurations dominated by low momentum components and it determines the infrared behavior of the solution. To illustrate this let us estimate the dual field action (3.16) for a field configuration that behaves as (3.21). We take $\rho$ to be a charge dipole with separation $L$. The main contribution to the action comes from the kinetic term in the infrared region,

$$
S_{\chi} \sim g^{2} m L^{2} \log V,
$$

where $V$ is the total volume (area) of the system. For the function (3.18) on the other hand the contributions of the kinetic and potential terms are of the same order and turn out to be

$$
S_{\chi} \sim z L^{2} \log V .
$$

This is much smaller than (3.23) and therefore the configuration (3.18) dominates the path integral over $\chi$.

The electric field of a pair of static charges thus does not decay exponentially in the minimally modified state, even though the vacuum does contain a dynamically generated 
scale. This leads us to suspect that the the minimally modified state is far from being the ground state in this sector. A calculation of the expectation value of the energy confirms this suspicion. The crucial terms are the last two terms, the vortex terms, in (3.14). To first order in $z$ the contribution of this term to the energy of the minimally modified state relative to the vacuum is

$$
\Delta E=m^{2} g^{2} \int d^{2} x[1-\cos (\theta \rho)]
$$

Far from the charges, we have $\theta \rho(x) \rightarrow L x_{1} / x^{2}$ when the dipole is parallel to the $x_{1}$ axis. The integral in (3.25) is positive and logarithmically divergent in the volume. It is hard to believe that this infrared divergent energy has anything to do with the actual interaction energy of the charges.

\section{The massless phase}

In the massless phase, the kinetic term of the dual field vanishes at zero momentum, $D^{-1}\left(k^{2}\right) \sim|k|$. The solution therefore can not be expanded in powers of $z D$ as before. The infrared behavior of the solution, however, can still be easily understood. It is determined by the potential term in (3.16) and is given by (3.21). The distance at which this behavior sets in is determined by the value of momentum at which the kinetic and potential terms are comparable, $x^{-1} \propto|k|=z / g^{2}$. The electric field is screened away from the dipole, decaying'g faster than $1 / x$. The "flux tube," carrying flux $g$ in a region of transverse size $g^{2} / z$, has energy

$$
\Delta E \sim z L .
$$

This is precisely the result of [5]. By itself it would suggest that the external charges are confined with the string tension $\sigma=z$, despite the absence of an area law for the spacelike Wilson loop. We suspect, however, that just as in the massive phase, this contradiction indicates that the minimally modified state provides a very poor bound on the energy of the charges.

A more reliable calculation of the interaction energy within the variational framework requires the introduction of additional variational parameters. This we do in the next section.

\section{THE INTERACTION ENERGY IN THE EXTENDED VARIATIONAL ANSATZ}

As we have seen in the previous section the minimally modified state has a prescribed profile of the electric field. This profile, as it turns out, does not conform to one's intuitive

\footnotetext{
${ }^{9}$ The screening here is not exponential but rather power-wise. This is due to the nonanalyticity of the $\chi$ propagator at zero momentum, $z \neq 0$ notwithstanding. Expanding $D^{-1}$ around $k=0$ we find that the leading term $D^{-1} \sim|k|$, gives in coordinate space $\langle\chi \chi\rangle \sim 1 / x^{3}$.
} 
picture of the profile of the electric field in the charged state. When there is a dynamically generated mass and an area law for the spacelike Wilson loop, one expects the electric field between the external charges to form a flux tube. In the minimally modified state the field is just the Coulomb field of an electric dipole. In the massless phase, conversely, we expect to find a dipole field, but instead we obtain a screened distribution similar to a flux tube. Moreover, the energy of the minimally modified state is unnaturally high.

\section{A. The extended ansatz}

The above leads us to believe that the minimally modified state is very far in its physical properties from the minimal energy state in the charged sector. It is apparent that the main

missing feature is freedom for the electric field to adjust its profile. We therefore introduce this additional variational freedom in our ansatz, writing

$$
\begin{aligned}
\psi[\mathbf{A}]= & \sum_{\left\{m_{\mathbf{n}^{\prime}}\right\}} \int\left[d \phi_{\mathbf{n}}\right] \exp \left[-\frac{1}{2} \sum_{\mathbf{r}, \mathbf{s}} A_{\mathbf{r} i}^{(\phi, m)} G^{-1}(\mathbf{r}-\mathbf{s}) A_{\mathbf{s} i}^{(\phi, m)}\right] \exp \left(i g \sum_{\mathbf{n}} \rho_{\mathbf{n}} \phi_{\mathbf{n}}\right) \\
& \times \exp \left(i \sum_{\mathbf{n}} \mathbf{e}_{\mathbf{n}} \cdot \mathbf{A}_{\mathbf{n}}^{(\phi, m)}\right) .
\end{aligned}
$$

We take the classical background field $\mathbf{e}$ to be transverse, $\nabla \cdot \mathbf{e}=0$, and we will treat it as an additional variational parameter in the charged sector.

Retracing the calculations of the previous sections we find that the normalization $Z$ is modified only in the vortex factor (3.9), wherein the external field $h_{\mathbf{r}^{\prime}}$ acting on the vortices becomes

$$
h=\frac{2 \pi}{g} \frac{1}{\nabla^{2}} \epsilon_{i j} \partial_{i} e_{j}+\theta \rho .
$$

The expectation value of the electric field is

$$
\left\langle E_{i}\right\rangle=g \frac{\partial_{i}}{\nabla^{2}} \rho-\frac{i \pi}{g} G^{-1} \frac{\epsilon_{i j} \partial_{j}}{\nabla^{2}}\langle m\rangle+e_{i} .
$$

[Note that e enters (4.3) as a simple shift and also as part of the external field (4.2) that determines $\langle m\rangle$.] The energy of this state is

$$
\begin{aligned}
\langle H\rangle=\frac{V}{4} \int & \frac{d^{2} k}{(2 \pi)^{2}}\left[G^{-1}(k)+k^{2} G(k)\right]-\frac{g^{2}}{2} \int d^{2} x \rho \frac{1}{\nabla^{2}} \rho \\
& +\frac{\pi^{2}}{2 g^{2}} \int d^{2} x d^{2} y \partial^{-2} G^{-2}(x-y)\langle m(x) m(y)\rangle \\
& -\frac{\alpha}{g^{2}} \int d^{2} x\left(\operatorname{Re}\left\langle e^{i \pi m(x)}\right\rangle-1\right) \\
& +\frac{i}{2} \zeta G^{-1}\langle m\rangle+\frac{1}{2} \int d^{2} x e^{2}
\end{aligned}
$$

where the penultimate term contains the potential $\zeta(x)$, defined via 


$$
e_{i}=\frac{g}{2 \pi} \epsilon_{i j} \partial_{j} \zeta
$$

Thus we have also

$$
h=-\zeta+\theta \rho
$$

The duality transformation now results in the following Lagrangian for the dual field $\chi$,

$$
L=g^{2} \chi D^{-1} \chi-2 z \int d^{2} x: \cos (\chi(x)+\zeta-\theta \rho):
$$

The correlation functions of $m$ are related to the correlation functions of $\chi$ via (3.15).

\section{B. The massive phase}

In the massive phase, we obtain as before to first order in $z$

$$
\begin{aligned}
\langle m(x)\rangle & =-2 i z \sin (\theta \rho(x)-\zeta(x)) \\
\langle m(x) m(y)\rangle & =2 z \cos (\theta \rho(x)-\zeta(x)) \delta^{2}(x-y) \\
\left\langle e^{i \pi m(x)}\right\rangle & =\exp [-4 z \cos (\theta \rho(x)-\zeta(x))]
\end{aligned}
$$

The e-dependent piece of the energy is

$$
\begin{aligned}
& \Delta E=m^{2} g^{2} \int d^{2} x[1-\cos (\theta \rho(x)-\zeta(x))] \\
& +z \int d^{2} x d^{2} y \zeta(x) G^{-1}(x-y) \sin (\theta \rho(y)-\zeta(y)) \\
& \quad-\frac{g^{2}}{8 \pi^{2}} \int d^{2} x \zeta \nabla^{2} \zeta
\end{aligned}
$$

The quantity (4.9) is to be minimized with respect to $\zeta$. It is obvious immediately that we will have $\zeta \rightarrow \theta \rho$ at large distances from the sources, so that the infrared divergence found in the minimally modified state will disappear. Noting that the second term is formally of order $g^{2}$ relative to the first, we drop it for now, subject to a consistency check at the end. The minimization equation for $\zeta$ then becomes very simple,

$$
\nabla^{2} \zeta-m^{2} \sin (\zeta-\theta \rho)=0
$$

To study (4.10) it is convenient to define $\tilde{\zeta}=\zeta-\theta \rho$ which satisfies the sine-Gordon equation with a singular source term,

$$
\nabla^{2} \tilde{\zeta}-m^{2} \sin \tilde{\zeta}=S
$$

The source term $S$ consists of a dipole layer along the line between the external point charges. For charges separated by a distance $L$, we have

$$
S(\mathbf{x})=2 \pi \delta^{\prime}\left(x_{2}\right) \eta\left(\frac{L}{2}+x_{1}\right) \eta\left(\frac{L}{2}-x_{1}\right)
$$


where $\eta$ is a step function. When $L$ is much larger than $1 / \mathrm{gm}$ the solution of (4.11) can be found in the region $-L / 2 \ll x_{1} \ll L / 2$. In this region $\tilde{\zeta}$ is approximately independent of $x_{1}$ and satisfies the one-dimensional sine-Gordon equation in $x_{2}$,

$$
\frac{\partial^{2} \tilde{\zeta}}{\partial x_{2}^{2}}-m^{2} \sin \tilde{\zeta}=S
$$

The singular source $S$ creates a discontinuity of $2 \pi$ at $x_{2}=0$. The solution for $x_{2}>0$ is hence half a sine-Gordon soliton, with $\tilde{\zeta}=\pi$ at $x_{2}=0+$ and $\tilde{\zeta} \rightarrow 0$ as $x_{2} \rightarrow \infty$. For $x_{2}<0$ it is half the antisoliton with $\tilde{\zeta}=-\pi$ at $x_{2}=0-$ and $\tilde{\zeta} \rightarrow 0$ as $x_{2} \rightarrow-\infty$. At distances greater than $1 / m$ from the $x_{1}$ axis, $\tilde{\zeta}$ vanishes exponentially. Therefore we have $\zeta \rightarrow \theta \rho$ exponentially. The vortex density $m(x)$ according to (4.8) vanishes exponentially. Referring to (4.3) we see that the total electric field vanishes exponentially outside the region of width $1 / m$, indeed forming a flux tube of thickness $1 / m$. This is perfectly in accord with our expectation for the field profile in a confining theory.

In (4.9) we see that the energy density of the flux tube is proportional to the energy of the sine-Gordon soliton solution with the proportionality coefficient $\frac{g^{2}}{4 \pi^{2}}$. The string tension therefore is

$$
\sigma=\frac{2}{\pi^{2}} g^{2} m
$$

This is again consistent with the result of the calculation of the spacelike Wilson loop in Section 2. We do get a slightly lower value for the string tension (a factor of $2 / \pi^{2}$ rather than $1 / 4$ ) here than we do from the spatial Wilson loop. This is a consequence of minimizing the energy independently in the charged sector.

\section{The massless phase}

The crucial feature of the massless phase is that now $D^{-1}$ vanishes at zero momentum. It is therefore incorrect to expand the classical solution for the dual equation of motion in powers of $z D$. Let us define $\chi_{c}$ to be the solution of the classical equation of motion for the dual field,

$$
g^{2} D^{-1} \chi_{c}+z \sin \left(\chi_{c}+\zeta-\theta \rho\right)=0 .
$$

For our purposes it will not be necessary to know the form of $\chi_{c}$ explicitly. The essential point is that unlike in the massive phase, for the $\chi$ propagator we must use the complete expression

$$
\langle\chi \chi\rangle_{\text {connected }}=\left[2 g^{2} D^{-1}+2 z \cos \left(\chi_{c}+\zeta-\theta \rho\right)\right]^{-1}
$$

without expanding in $z$. Calculating the correlation functions of the vorticity via (3.15), and substituting into (4.4), we obtain for the energy

$$
\begin{aligned}
& \Delta E=-\frac{g^{2}}{8 \pi^{2}}\left(\chi_{c}+\zeta\right) \nabla^{2}\left(\chi_{c}+\zeta\right) \\
& +4 g^{4}\left(\frac{\pi^{2}}{2 g^{2}}-\frac{2 \alpha}{g^{2}}\right) \operatorname{Tr} D^{-1}\left\{\left[2 g^{2} D^{-1}+2 z \cos \left(\chi_{c}+\zeta-\theta \rho\right)\right]^{-1}-\left[2 g^{2} D^{-1}+2 z\right]^{-1}\right\} D^{-1} .
\end{aligned}
$$


To find the infrared asymptotics we neglect $D^{-1}$ in the denominators in (4.17) since it vanishes at zero momentum. The second term then becomes

$$
\int d^{2} x \nabla^{2}\left(\frac{1}{\cos \left(\chi_{c}+\zeta-\theta \rho\right)}\right) \text {. }
$$

This is the integral of a total derivative and it vanishes since $1 / \cos \left(\chi_{c}+\zeta-\theta \rho\right)$ does not diverge at infinity.

The profile of $\zeta$ is therefore determined by minimizing the first term in (4.17). The solution is

$$
\chi_{c}+\zeta=0
$$

Thus at distances larger than $g^{2} / z$, the induced electric field $\frac{g}{2 \pi} \epsilon_{i j} \partial_{j} \zeta$ cancels the contribution of the vortices $\frac{g}{2 \pi} \epsilon_{i j} \partial_{j} \chi_{c}$. The total electric field at large distances is therefore just the field of the electric dipole. This is again consistent with our expectation for the behavior of electric field in a massless phase.

\section{DISCUSSION}

In this paper we studied compact $\mathrm{QED}_{3}$ with mixed action in the gauge invariant Gaussian variational approach. We find that the theory has two phases. When $\beta$, the coefficient of the charge- 2 term in the action, is not too negative the theory is massive and confining. For $\beta$ large and negative no mass is generated and the theory is not confining. We have also calculated variationally the interaction energy of a state with two external charges.

The authors of [5] studied a minimally modified variational state with no dynamical mass within the framework of the pure fundamental theory. As emerges from our analysis the vacuum of the pure fundamental theory is massive. This mass has to be introduced explicitly in the Gaussian. The nonvanishing string tension is due to this dynamical mass and behaves according to the Polyakov formula $\sigma \sim g^{2} m \sim z^{1 / 2}$ where $z$ is the vortex fugacity.

The massless state is sensible in the theory with mixed action for $\alpha>\pi^{2} / 4$. This phase is not confining. The calculation of [5], however, yields a string tension $\sigma \sim z$ which is nonvanishing, though parametrically smaller than in the massive phase. We now understand that this nonvanishing string tension is an artifact of the minimally modified state. A better variational state does not exhibit flux tube formation in the massless phase.

The main lesson we have learned is that to get a good estimate for the interaction energy of external charges we have to introduce a background electric field as an additional variational parameter. The minimally modified state of [11, 12] which does not allow for this variation is quite poor energetically. It has a wrong profile of the electric field and as a consequence overestimates significantly the interaction energy. We expect the same to be true in nonabelian gauge theories, so we expect the calculation of the interaction energy of external charges there to be more complicated than envisioned in [11, 12, .

On the positive side we found that the calculation of the spacelike Wilson loop gives a good estimate for the interaction potential and the associated string tension even though the variational wave function, and indeed the cutoff Hamiltonian theory, is not Lorentz invariant. 


\section{ACKNOWLEDGMENTS}

A. K. thanks the Department of Nuclear Physics at Tel Aviv University for their hospitality. B. S. thanks the Theoretical Physics group at Oxford University for hospitality and PPARC for financial support during his visit there. He also thanks the Weizmann Institute of Science for its continuing hospitality. A. K. is supported by a PPARC Advanced Fellowship. The work of B. S. is partially supported by the Israel Science Foundation under Grant No. 255/96-1. 


\section{REFERENCES}

[1] A. M. Polyakov, Phys. Lett. 59B, 82 (1975); Nucl. Phys. B 120, 429 (1977).

[2] C. G. Callan, R. Dashen and D. J. Gross, Phys. Rev. D 17, 2717 (1978); ibid. 19, 1826 (1979).

[3] T. Banks, R. Myerson and J. Kogut, Nucl. Phys. B 129, 493 (1977).

[4] M. Creutz, Phys. Rev. D 21, 2308 (1980).

[5] S. D. Drell, H. R. Quinn, B. Svetitsky, and M. Weinstein, Phys. Rev. D 19, 619 (1979).

[6] I. Kogan and A. Kovner, Phys. Rev. D 52, 3719 (1995).

[7] C. Heinemann, C. Martin, D. Vautherin, and E. Iancu, hep-ph/9802036.

[8] I. Kogan and A. Kovner, Phys. Rev. D 51, 1948 (1995).

[9] W. E. Brown and I. I. Kogan, hep-th/9705136.

[10] W. E. Brown, hep-th/9711189.

[11] K. Zarembo, Phys. Lett. B 421, 325 (1998); hep-th/9806150.

[12] D. Diakonov, hep-th/9805137.

[13] T. R. Morris, Phys. Rev. D 53, 7250 (1996).

[14] S. Ben-Menahem, Phys. Rev. D 20, 1923 (1979).

[15] J. Ambjorn and J. Greensite, J. High Energy Phys. 05 (1998) 004, hep-lat/9804022. 